\title{
BOUNDED COMPLEXES OF PERMUTATION MODULES
}

\author{
DAVID J. BENSON AND JON F. CARLSON
}

(Communicated by Sarah Witherspoon)

\begin{abstract}
Let $k$ be a field of characteristic $p>0$. For $G$ an elementary abelian $p$-group, there exist collections of permutation modules such that if $C^{*}$ is any exact bounded complex whose terms are sums of copies of modules from the collection, then $C^{*}$ is contractible. A consequence is that if $G$ is any finite group whose Sylow $p$-subgroups are not cyclic or quaternion, and if $C^{*}$ is a bounded exact complex such that each $C^{i}$ is a direct sum of one dimensional modules and projective modules, then $C^{*}$ is contractible.
\end{abstract}

\section{INTRODUCTION}

The study of complexes of permutation modules for finite groups has a long history. They occur naturally in the study of group actions on CW-complexes and manifolds; see for example Bredon [2], Illman 3]. Of particular interest are the complexes arising from collections of subgroups in the work of Quillen [4, Webb [6, 7] and others. They also arise in the theory of splendid equivalences between derived categories of blocks, in the work of Rickard [5].

In this paper we examine bounded exact complexes of permutation modules, and find conditions which force them to be contractible. Of course for a cyclic group $G \cong \mathbb{Z} / p$ in characteristic $p$ there exist bounded exact complexes that are not contractible, such as the periodicity complex $0 \rightarrow k \rightarrow k G \rightarrow k G \rightarrow k \rightarrow 0$, so the game is to find conditions prohibiting examples constructed from these.

Suppose that $\mathcal{S}$ is a collection of modules over the group algebra $k G$ of a finite group $G$ with coefficients in a field $k$ of characteristic $p>0$. We investigate the question of what collections $\mathcal{S}$ exist with the property that any bounded exact complex of modules in the additive subcategory $\operatorname{Add}(\mathcal{S})$ is contractible. We show that if $G$ is an elementary abelian $p$-group having rank at least two, then there are collections of permutation modules that satisfy this property. In addition, for any finite group having $p$-rank at least two, the collection of all modules that are either projective or have dimension one has the property. There are many more such collections. Our main theorem is Theorem 3.1 which gives a sufficient condition on a collection of subgroups of an elementary abelian group for every bounded exact complex with these stabilisers to be contractible. It is easy to see, by inflating and inducing up the periodicity complex for a cyclic subquotient, that this condition is also necessary. The proof of the main theorem involves a spectral

Received by the editors December 6, 2020, and, in revised form, June 22, 2021, and September 3,2021 .

2020 Mathematics Subject Classification. Primary 20J06, 20C20.

Key words and phrases. Finite groups, permutation modules, bounded exact complex.

The second author was partially supported by Simons Foundation Grant 054813-01. This work was supported by EPSRC grant number EP/R014604/1. 
sequence argument and some commutative algebra. As an application of the main theorem, we show that for a finite group of $p$-rank at least two, every bounded exact complex of sums of projective modules and one dimensional modules is contractible, in contrast with the cyclic case discussed above.

A recent paper by Paul Balmer and the first author is something of a complement to the results presented here. In [1, it is proved that every module over the modular group algebra of an elementary abelian $p$-group has a finite resolution by permutation modules. By splicing together left and right resolutions obtained in this way, we obtain large supplies of exact complexes of permutation modules that do not split.

\section{PRELIMINARIES}

Let $k$ be a field of characteristic $p$. Let $G$ be an elementary abelian $p$-group of rank $r$. That is, $G \cong C_{p}^{r}$ is a direct product of $r$ copies of the cyclic group $C_{p}$ of order $p$.

Recall that if $p=2$, then the cohomology ring of $G, \mathrm{H}^{*}(G, k) \cong \operatorname{Ext}_{k G}^{*}(k, k)$ is a polynomial ring

$$
\mathrm{H}^{*}(G, k)=k\left[x_{1}, \ldots, x_{r}\right]
$$

with every $x_{i}$ having degree one. If $p$ is odd, then

$$
\mathrm{H}^{*}(G, k)=k\left[x_{1}, \ldots, x_{r}\right] \otimes \Lambda\left(u_{1}, \ldots, u_{r}\right),
$$

where $\Lambda$ is the exterior algebra generated by elements $u_{i}$ is degree one, and every $x_{i}$ has degree 2 .

If $M$ is a finitely generated $k G$-module, then $\mathrm{H}^{*}(G, M)$ is a finitely generated module over $\mathrm{H}^{*}(G, k)$. For $E$ a subgroup of $G$, let $k G / E$ denote the permutation module on the cosets of $E$ in $G$. By Frobenius Reciprocity, or the Eckmann-Shapiro Lemma, we have an isomorphism of $\mathrm{H}^{*}(G, k)$-modules $\mathrm{H}^{*}(G, k G / E) \cong \mathrm{H}^{*}(E, k)$, with the action of $\mathrm{H}^{*}(G, k)$ on $\mathrm{H}^{*}(E, k)$ given by the restriction map $\mathrm{H}^{*}(G, k) \rightarrow$ $\mathrm{H}^{*}(E, k)$.

For the proof of the main theorem of the paper we require some technical facts. The first is easily verified by the reader.

Lemma 2.1. Let $R$ be a ring and $u \in R$.

(i) If $N \subseteq M$ are $R$-modules, and $u N=0=u(M / N)$, then $u^{2} M=0$.

(ii) If $M$ and $N$ are $R$-modules, $u M=0$, and $u$ is regular on $N$, then $\operatorname{Hom}_{R}(M, N)=0$.

Theorem 2.2. Let $G$ be an elementary abelian p-group of rank $r$. Let $s \leqslant r$, and let $M, N$ be finite direct sums of copies of $\mathrm{H}^{*}(G, k)$-modules of the form $\mathrm{H}^{*}(E, k)$ where the $E$ 's are subgroups of rank s. Let $M_{0} \subseteq M, N_{0} \subseteq N$ be graded $\mathrm{H}^{*}(G, k)$-submodules and let $X=M / M_{0}$. Suppose that there are elements $u$ and $v$ in $\mathrm{H}^{*}(G, k)$ such that $u$ and $v$ annihilate $X$ but $u, v$ is a regular sequence on $N$. Then

(i) $\operatorname{Hom}_{\mathrm{H}^{*}(G, k)}(X, N)=0$,

(ii) $\operatorname{Ext}_{\mathrm{H}^{*}(G, k)}^{1}(X, N)=0$,

(iii) $\operatorname{Hom}_{\mathrm{H}^{*}(G, k)}(M, N) \rightarrow \operatorname{Hom}_{\mathrm{H}^{*}(G, k)}\left(M_{0}, N\right)$ is an isomorphism,

(iv) $\operatorname{Hom}_{\mathrm{H}^{*}(G, k)}\left(M_{0}, N_{0}\right) \rightarrow \operatorname{Hom}_{\mathrm{H}^{*}(G, k)}\left(M_{0}, N\right)$ is injective, and

(v) $\operatorname{Hom}_{\mathrm{H}^{*}(G, k)}\left(M_{0}, N_{0}\right)$ is zero in negative degrees, that is, there are no homomorphisms that lower degree. 
Proof. To prove (i) and (ii), we use a standard depth argument from commutative algebra. Choose elements $u, v \in \mathrm{H}^{*}(G, k)$ such that $u$ and $v$ annihilate $X$, but form a regular sequence on $N$. Consider the exact sequence

$$
0 \longrightarrow N \stackrel{u}{\longrightarrow} N \longrightarrow N / u N \longrightarrow 0 \text {. }
$$

From this we get a long exact sequence

$$
\begin{gathered}
0 \longrightarrow \operatorname{Hom}_{\mathrm{H}^{*}(G, k)}(X, N) \stackrel{u}{\longrightarrow} \operatorname{Hom}_{\mathrm{H}^{*}(G, k)}(X, N) \longrightarrow \operatorname{Hom}_{\mathrm{H}^{*}(G, k)}(X, N / u N) \\
\longrightarrow \operatorname{Ext}_{\mathrm{H}^{*}(G, k)}^{1}(X, N) \stackrel{u}{\longrightarrow} \operatorname{Ext}_{\mathrm{H}^{*}(G, k)}^{1}(X, N) \longrightarrow \cdots
\end{gathered}
$$

Since $u$ annihilates $X$, it annihilates both $\operatorname{Hom}_{\mathrm{H}^{*}(G, k)}(X, N)$ and $\operatorname{Ext}_{\mathrm{H}^{*}(G, k)}^{1}(X, N)$. Thus $\operatorname{Hom}_{\mathrm{H}^{*}(G, k)}(X, N)=0$. By a similar argument, because $v$ is regular on $N / u N$, we have that $\operatorname{Hom}_{\mathrm{H}^{*}(G, k)}(X, N / u N)=0$. From this it follows that $\operatorname{Ext}_{\mathrm{H}^{*}(G, k)}^{1}(X, N)$ $=0$ as asserted.

From the exact sequence

$$
0 \longrightarrow M_{0} \longrightarrow M \longrightarrow X \longrightarrow 0
$$

we obtain an exact sequence

$$
\begin{aligned}
0 & \rightarrow \operatorname{Hom}_{\mathrm{H}^{*}(G, k)}(X, N) \rightarrow \operatorname{Hom}_{\mathrm{H}^{*}(G, k)}(M, N) \\
& \rightarrow \operatorname{Hom}_{\mathrm{H}^{*}(G, k)}\left(M_{0}, N\right) \rightarrow \operatorname{Ext}_{\mathrm{H}^{*}(G, k)}^{1}(X, N) \rightarrow \cdots .
\end{aligned}
$$

Thus (iii) follows from (i) and (ii).

Part (iv) follows from the fact that the map $N_{0} \rightarrow N$ is injective. To prove part (v), we note that $\operatorname{Hom}_{\mathrm{H}^{*}(G, k)}(M, N)$ is zero in negative degrees, so by (iii) the same is true of $\operatorname{Hom}_{\mathrm{H}^{*}(G, k)}\left(M_{0}, N\right)$, and then by (iv) the same is true of $\operatorname{Hom}_{\mathrm{H}^{*}(G, k)}\left(M_{0}, N_{0}\right)$.

The next result is useful in the proof of our main theorem.

Lemma 2.3. Suppose that $\left(C^{*}, \delta^{*}\right)$ is a cochain complex in some abelian category, and that for some $n$ we have direct sum decompositions

$$
C^{n} \cong U^{n} \oplus V^{n} \quad \text { and } \quad C^{n+1} \cong U^{n+1} \oplus V^{n+1} .
$$

If the composite $a: U^{n} \longmapsto C^{n} \stackrel{\delta^{n}}{\longrightarrow} C^{n+1} \rightarrow U^{n+1}$ is an isomorphism then $C^{*}$ has a contractible subcomplex $D^{*}: 0 \rightarrow U^{n} \stackrel{a}{\rightarrow} U^{n+1} \rightarrow 0$. Moreover the sequence

$$
0 \longrightarrow D^{*} \longrightarrow C^{*} \longrightarrow(C / D)^{*} \longrightarrow 0
$$

of cochain maps splits.

Proof. If the map $a: U^{n} \rightarrow U^{n+1}$ is invertible then we have a commutative diagram

$D^{*}:$

$C^{*}$ :

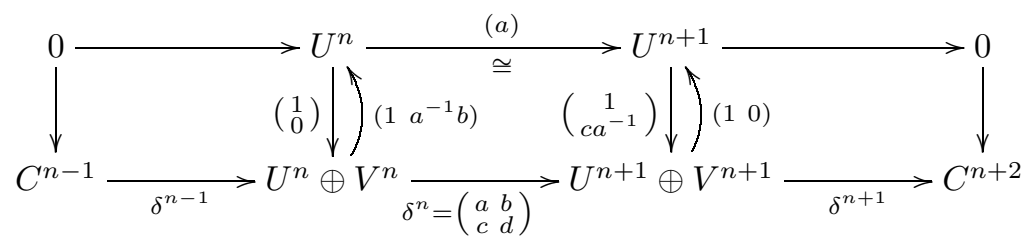




\section{THE MAIN THEOREM}

Our objective in this section is to prove the main theorem of the paper. If $\mathcal{S}$ is a collection of finitely generated $k G$-modules, then $\operatorname{Add}(\mathcal{S})$ is the full additive subcategory of the module category consisting of all $k G$-modules that are isomorphic to finite direct sums of copies of objects in $\mathcal{S}$.

Theorem 3.1. Let $G$ be an elementary abelian p-group and $k$ a field of characteristic p. Suppose that $\mathcal{H}$ is a collection of subgroups of $G$ with the property that there is no pair $E, F$ of elements in $\mathcal{H}$ such that $E \subseteq F$ with index $p$. If

$$
0 \longrightarrow C^{0} \longrightarrow C^{1} \longrightarrow \cdots \longrightarrow C^{\ell} \longrightarrow 0
$$

is a bounded exact sequence of $k G$-modules in $\operatorname{Add}(\{k G / E \mid E \in \mathcal{H}\})$ then $C^{*}$ is contractible.

Before beginning the proof, we present Lemma 3.2 which is needed in an essential case of the proof.

Lemma 3.2. Let $s^{\prime}<s \leqslant r=\operatorname{rank}(G)$. Then the sequence of Dickson invariants

$$
c_{r, r-s}, c_{r, r-s+1}, \ldots, c_{r, r-s^{\prime}-1} \in H^{*}(G, k)
$$

restricts to a regular sequence of length $s-s^{\prime}$ on every subgroup of $G$ of rank $s$, and restricts to zero on every subgroup of rank at most $s^{\prime}$.

Proof. In the statement of this lemma, we are using the polynomial subring of $H^{*}(G, k)$ generated by the Bocksteins $\beta(y) \in H^{2}\left(G, \mathbb{F}_{p}\right) \subseteq H^{2}(G, k)$ of the elements $y \in H^{1}\left(G, \mathbb{F}_{p}\right)$. For every subgroup of $G$, the restriction map is surjective on these polynomial rings, with kernel generated by its degree two elements.

The group Aut $(G) \cong \mathrm{GL}\left(r, \mathbb{F}_{p}\right)$ acts on this polynomial subring, and the invariants of this action form a polynomial ring on the Dickson invariants $k\left[c_{r, 0}, \ldots, c_{r, r-1}\right]$ over which $H^{*}(G, k)$ is a finitely generated free module. In particular, the Dickson invariants form a regular sequence of length $r$ in $H^{*}(G, k)$. The degree of $c_{r, i}$ is $2\left(p^{r}-p^{i}\right)$.

Recall from Wilkerson [8] that the Dickson invariants $c_{r, i}$ are defined by the equation

$$
\prod_{y \in H^{1}\left(G, \mathbb{F}_{p}\right)}(T-\beta(y))=T^{p^{r}}+\sum_{i=0}^{r-1}(-1)^{r-i} c_{r, i} T^{p^{i}},
$$

where $T$ is an indeterminate. By proposition $1.3(\mathrm{~b})$ of that paper, the restriction of $c_{r, r-i}$ to a rank $s$ subgroup is the element $c_{s, s-i}^{p^{r-s}}$ of that cohomology ring if $i \leqslant s$ and zero if $i>s$. Thus the given sequence restricts to $c_{s, 0}^{p^{r-s}}, c_{s, 1}^{p^{r-s}}, \ldots, c_{s, s-s^{\prime}-1}^{p^{r-s}}$ on a subgroup of rank $s$, and to zero on a subgroup of rank at most $s^{\prime}$.

We are now prepared to prove our theorem.

Proof of Theorem 3.1. Suppose that $C^{*}$ is an exact complex of permutation modules as in the statement of Theorem 3.1. Without loss of generality, we may assume that $C^{*}$ does not have a nontrivial direct summand that is contractible. Let

$$
\text { .. } \longrightarrow P_{2} \longrightarrow P_{1} \longrightarrow P_{0} \longrightarrow k \longrightarrow 0
$$


be a projective resolution of $k$, the trivial $k G$-module. We consider the two spectral sequences whose $E_{0}$-term is the double complex

$$
E_{0}^{i, j}=\operatorname{Hom}_{k G}\left(P_{j}, C^{i}\right) .
$$

If we first take the differential that comes from the complex $C^{*}$, then we get that $E_{1}^{*, *}$ is identically zero. This is because $P_{j}$ is projective and the complex $C^{*}$ is exact.

It follows that the spectral sequence obtained by first starting with the differential on $P_{*}$ converges to zero. Indeed, it converges to zero after a finite number of steps, since there are only a finite number of columns. The $E_{1}$ term has the form

$$
E_{1}^{i, j}=\mathrm{H}^{j}\left(G, C^{i}\right) \Longrightarrow 0,
$$

and all differentials on this and subsequent pages are homomorphisms of $\mathrm{H}^{*}(G, k)$ modules. Let $d_{i}$ denote the differential on the $E_{i}$ page of this spectral sequence. Note that $d_{1}$ is not the zero map as otherwise it is impossible for the spectral sequence to converge to zero. Each $C^{i}$ is a direct sum of permutation modules on subgroups $E \in \mathcal{H}$. The corresponding summand of $\mathrm{H}^{*}\left(G, C^{i}\right)$ is $\mathrm{H}^{*}(G, k G / E) \cong$ $\mathrm{H}^{*}(E, k)$. Note that the larger the subgroup the smaller is the dimension of the permutation module, and the larger is the Krull dimension of its cohomology.

Let $s$ be the maximum $p$-rank of any element of $\mathcal{H}$. If $s=0$ then $C^{*}$ is a finite exact complex of projectives, which is therefore contractible. Suppose next that $s=1$. Then each subgroup in $\mathcal{H}$ is cyclic, and not trivial, by the hypothesis. For distinct cyclic subgroups $E$ and $F$, there are no nonzero $\mathrm{H}^{*}(G, k)$-module homomorphisms from $\mathrm{H}^{*}(G, k(G / E))$ to $\mathrm{H}^{*}(G, k(G / F))$, since we can find an element of $\mathrm{H}^{2}(G, k)$ that annihilates $\mathrm{H}^{*}(G, k(G / E))$ but acts regularly on $\mathrm{H}^{*}(G, k(G / F))$. So the only way to have $d_{1} \neq 0$ is if for some $i$, both $C^{i}$ and $C^{i+1}$ have direct summands isomorphic to $k(G / E)$ for some $E \in \mathcal{H}$, and the composition $k(G / E) \rightarrow C^{i} \rightarrow C^{i+1} \rightarrow k(G / E)$ is an isomorphism because it induces an isomorphism $\mathrm{H}^{0}(G, k(G / E)) \rightarrow \mathrm{H}^{0}(G, k(G / E)$ ) (a self-map which is an isomorphism on the socle is an isomorphism). This cannot happen, since by Lemma 2.3 it violates the assumption that $C^{*}$ has no contractible direct summands, so $d_{1}=0$. But also $d_{n}=0$ for $n \geqslant 2$, since there are no homomorphisms between the modules $\mathrm{H}^{*}(G, E)$ that lower degrees. This implies that the spectral sequence cannot converge to zero, and so the theorem is proved in this case. So from now on, we assume that $s \geqslant 2$.

Let $\mathcal{H}^{\prime}$ be the set of subgroups in $\mathcal{H}$ that have rank $s$, and let $\mathcal{H}^{\prime \prime}=\mathcal{H} \backslash \mathcal{H}^{\prime}$. We decompose each $C^{i}$ as $D^{i} \oplus U^{i}$, where $D^{i}$ is a direct sum of permutation modules $k(G / E)$ with $E \in \mathcal{H}^{\prime}$ having rank $s$, and where $U^{i}$ is a direct sum of permutation modules on cosets of subgroups in $\mathcal{H}^{\prime \prime}$. By Lemma 3.2 with $s^{\prime} \leqslant s-2$, there are homogeneous elements $u, v \in \mathrm{H}^{*}(G, k)$ which form a regular sequence on every $\mathrm{H}^{*}\left(G, D^{i}\right)$ but annihilate all $\mathrm{H}^{*}\left(G, U^{i}\right)$. In particular, since $u$ is regular on $\mathrm{H}^{*}\left(G, D^{i}\right)$ but annihilates $\mathrm{H}^{*}\left(G, U^{i}\right)$, there are no nonzero $\mathrm{H}^{*}(G, k)$-module homomorphisms from $\mathrm{H}^{*}\left(G, U^{i}\right)$ to $\mathrm{H}^{*}\left(G, D^{i+1}\right)$. Hence, $d_{1}\left(\mathrm{H}^{*}\left(G, U^{*}\right)\right) \subseteq \mathrm{H}^{*}\left(G, U^{*}\right)$ and we obtain a short exact sequence of complexes

$$
0 \longrightarrow \mathrm{H}^{*}\left(G, U^{*}\right) \longrightarrow E_{1}^{*, *} \stackrel{\vartheta_{1}}{\longrightarrow} \mathrm{H}^{*}\left(G, D^{*}\right) \longrightarrow 0 .
$$

Note that

$$
E_{1}^{*, *} / \mathrm{H}^{*}\left(G, U^{*}\right)=\mathrm{H}^{*}\left(G, C^{*}\right) / \mathrm{H}^{*}\left(G, U^{*}\right) \cong \mathrm{H}^{*}\left(G, C^{*} / U^{*}\right) \cong \mathrm{H}^{*}\left(G, D^{*}\right) .
$$


We point out that the above is something of an abuse of notation. In particular, $D^{*}$ is not a complex. However, $\mathrm{H}^{*}\left(G, D^{*}\right)$ is a complex of $\mathrm{H}^{*}(G, k)$-modules when regarded as a quotient in (3.1). The differential $\hat{d}_{1}$ on $\mathrm{H}^{*}\left(G, D^{*}\right)$ is induced from $d_{1}$ as in the quotient.

Our aim is to prove that if $C^{*}$ is not a contractible complex, then the above spectral sequence cannot converge to zero, thus giving us a contradiction. Again, we are assuming that $C^{*}$ has no contractible direct summands. We proceed by induction on the pages of the spectral sequence. The induction statement is the following.

Induction Statement for page $n$. There exist homogeneous elements $u_{n}, v_{n}$ in $\mathrm{H}^{*}(G, k)$ and, for every $i, j$, there is a $k$-subspace $\mathcal{U}_{n}^{i, j} \subseteq E_{n}^{i, j}$ such that the following hold.

$\mathcal{I S}(n, 1): \quad \mathcal{U}_{n}^{i, *}$ is an $\mathrm{H}^{*}(G, k)$-submodule of $E_{n}^{i, *}$. Let $\mathcal{D}_{n}^{i, j}=E_{n}^{i, j} / \mathcal{U}_{n}^{i, j}$.

$\mathcal{I S}(n, 2): u_{n}, v_{n}$ form a regular sequence on $\mathrm{H}^{*}\left(G, D^{*}\right)$.

$\mathcal{I S}(n, 3): \quad u_{n}, v_{n}$ annihilate $\mathcal{U}_{n}^{*, *}$.

$\mathcal{I S}(n, 4): \quad$ There are natural inclusions $\mathcal{D}_{n}^{i, j} \subseteq \mathcal{D}_{n-1}^{i, j} \subseteq \cdots \subseteq \mathcal{D}_{1}^{i, j}=\mathrm{H}^{j}\left(G, D^{i}\right)$ preserved by the action of $\mathrm{H}^{*}(G, k)$ increasing the $j$ coordinate. Moreover, $\mathrm{H}^{*}\left(G, D^{*}\right) / \mathcal{D}_{n}^{*, *}$ is annihilated by both $u_{n}$ and $v_{n}$.

$\mathcal{I S}(n, 5): \quad \mathcal{U}_{n}^{*, *}$ is a subcomplex of $\left(E_{n}^{*, *}, d_{n}\right)$ so that

$$
0 \longrightarrow \mathcal{U}_{n}^{*, *} \longrightarrow E_{n}^{*, *} \stackrel{\vartheta_{n}}{\longrightarrow} \mathcal{D}_{n}^{*, *} \longrightarrow 0
$$

is an exact sequence of complexes of $\mathrm{H}^{*}(G, k)$-modules. We write $\hat{d}_{n}$ for the differential on $\mathcal{D}_{n}^{*, *}$ induced by the differential $d_{n}$ on $E_{n}^{*, *}$.

$\mathcal{I S}(n, 6): \quad d_{n}\left(E_{n}^{i, j}\right) \subseteq \mathcal{U}_{n}^{i+n, j-n+1}$ for all $i$ and $j$.

We begin the induction with $n=1$. Let $\mathcal{U}_{1}^{i, j}=\mathrm{H}^{j}\left(G, U^{i}\right), \mathcal{D}_{1}^{i, j}=\mathrm{H}^{j}\left(G, D^{i}\right)$, $u_{1}=u, v_{1}=v$. Then conditions $\mathcal{I} \mathcal{S}(1,1)$ and $\mathcal{I} \mathcal{S}(1,5)$ have already been proved. Conditions $\mathcal{I S}(1,2)$ and $\mathcal{I S}(1,3)$ are true by the choice of $u$ and $v$. Condition $\mathcal{I S}(1,4)$ is obvious. The only remaining task is to verify $\mathcal{I S}(1,6)$ which is easily seen to be equivalent to the condition that the map induced via the quotient (3.1)

$$
\hat{d}_{1}: \mathcal{D}_{1}^{i, *} \cong \mathrm{H}^{*}\left(G, D^{i}\right) \longrightarrow \mathcal{D}_{1}^{i+1, *} \cong \mathrm{H}^{*}\left(G, D^{i+1}\right)
$$

is the zero map for all $i=0, \ldots, \ell-1$. This is a degree zero map of $\mathrm{H}^{*}(G, k)$-modules induced by the coboundary map on $C^{*}$. But note that if $E, F \in \mathcal{H}^{\prime}$ and $E \neq F$, then there are no nonzero homomorphisms from $\mathrm{H}^{*}(G, k(G / E))$ to $\mathrm{H}^{*}(G, k(G / F))$, for the same reason as in the case $s=1$. Consequently, the only way that $\hat{d}_{1}$ could be nonzero is if, for some $i, C^{i}$ and $C^{i+1}$ both had direct summands isomorphic to $k(G / E)$ for some $E \in \mathcal{H}^{\prime}$, and the composition of the injection of one followed by the coboundary followed by the projection onto the other is an isomorphism. However, such a situation does not occur because by Lemma 2.3 it violates our assumption that the complex $C^{*}$ has no contractible direct summands. Thus, the induction statement is true in the case that $n=1$.

Now we assume for some $n \geqslant 1$ that the Induction Statement for page $n$ is true. We need to show that it holds also for page $n+1$. Let $u_{n+1}=u_{n}^{2}$ and $v_{n+1}=v_{n}^{2}$. Note that $\mathcal{I S}(n+1,2)$ is automatic from $\mathcal{I S}(n, 2)$. Writing $K^{i, j}$ for the kernel of $d_{n}$ on $E_{n}^{i, j}$, recall that $E_{n+1}^{i, j}$ is the quotient of $K^{i, j}$ by the submodule $d_{n}\left(E_{n}^{i-n, j+n-1}\right)$. Let $\mathcal{D}_{n+1}^{i, j}=\vartheta_{n}\left(K^{i, j}\right)$, the image in $\mathcal{D}_{n}^{i, j}$ of the kernel of $d_{n}$ on $E_{n}^{i, j}$. By $\mathcal{I} \mathcal{S}(n, 6)$, we 
have that $\vartheta_{n}\left(d_{n}\left(E_{n}^{i-n, j+n-1}\right)\right)=0$. Hence there is a well defined homomorphism $\vartheta_{n+1}$, and an exact sequence

$$
0 \longrightarrow \mathcal{U}_{n+1}^{*, *} \longrightarrow E_{n+1}^{*, *} \stackrel{\vartheta_{n+1}}{\longrightarrow} \mathcal{D}_{n+1}^{*, *} \longrightarrow 0 .
$$

That is, we define $\mathcal{U}_{n+1}^{*, *}$ to be the kernel of $\vartheta_{n+1}$, which is the map induced by $\vartheta_{n}$, and $\hat{d}_{n+1}$ to be the differential induced on $\mathcal{D}_{n+1}^{*, *}$ by the differential $d_{n+1}$ on $E_{n+1}^{*, *}$ via the above sequence.

Because $\vartheta_{n}$ is an $\mathrm{H}^{*}(G, k)$-homomorphism and both the kernel and image of $d_{n}$ are $\mathrm{H}^{*}(G, k)$-submodules, we have that $\vartheta_{n+1}$ is also an $\mathrm{H}^{*}(G, k)$-homomorphism. This proves $\mathcal{I S}(n+1,1)$. Condition $\mathcal{I S}(n+1,3)$ is a consequence of the fact that $\mathcal{U}_{n+1}^{*, *}$ is a quotient of $\mathcal{U}_{n}^{*, *}$ by a submodule thereof. So it is annihilated by $u_{n}$ and $v_{n}$. For condition $\mathcal{I} \mathcal{S}(n+1,4)$, notice that if $x \in \mathcal{D}_{n}^{i, j}$, then $x=\vartheta_{n}(y)$ for some $y$ in $E_{n}^{i, j}$. By $\mathcal{I S}(n, 6)$ and $\mathcal{I S}(n, 2), d_{n}(y) \in \mathcal{U}_{n}^{i+n, j-n+1}$ is annihilated by $u_{n}$ and $v_{n}$. That is, $u_{n} y$ and $v_{n} y$ are in the kernel of $d_{n}$. So $\vartheta_{n}\left(u_{n} y\right)=u_{n} x$ and $\vartheta_{n}\left(v_{n} y\right)=v_{n} x$ are in $\mathcal{D}_{n+1}^{i, j}$. Thus, $\mathcal{D}_{n}^{*, *} / \mathcal{D}_{n+1}^{*, *}$ is annihilated by $u_{n}$ and $v_{n}$. It follows from $\mathcal{I} \mathcal{S}(n, 4)$ and Lemma 2.1 that $\mathrm{H}^{*}\left(G, D^{*}\right) / \mathcal{D}_{n+1}^{*, *}$ is annihilated by $u_{n+1}$ and $v_{n+1}$.

We can see that there are no nonzero homomorphisms from $\mathcal{U}_{n+1}^{*, *}$ to $\mathcal{D}_{n+1}^{*, *}$. The reason is that by $\mathcal{I S}(n+1,2)$ and $\mathcal{I S}(n+1,4), u_{n+1}$ is a regular element on $\mathcal{D}_{n+1}^{*, *}$, while it annihilates $\mathcal{U}_{n+1}^{*, *}$ by $\mathcal{I} \mathcal{S}(n+1,3)$ and Lemma 2.1. This proves $\mathcal{I S}(n+1,5)$.

Finally, we observe that $\hat{d}_{n+1}\left(\mathcal{D}_{n+1}^{*, *}\right)=0$. The reason for this is that $\mathcal{D}_{n+1}^{*, *}$ is an $\mathrm{H}^{*}(G, k)$-submodule of $\mathrm{H}^{*}\left(G, D^{*}\right)$, and the homomorphism $\hat{d}_{n+1}$, which lowers the degrees, is the zero map by Theorem 2.2(v). This implies that $d_{n+1}\left(E_{n+1}^{*, *}\right) \subseteq \mathcal{U}_{n+1}^{*, *}$. Consequently, Condition $\mathcal{I S}(n+1,6)$ holds.

Thus, we have shown that the Induction Statement for page $n$ of the spectral sequence implies that of page $n+1$. Hence, the statement holds for all pages. Because the complex $C^{*}$ has only $\ell+1$ nonzero terms, the spectral sequence has only $\ell+1$ nonzero columns, and it must stop after $\ell+2$ steps. That is, $E_{\ell+2}^{*, *}=E_{\infty}^{*, *}=\{0\}$. However, this is a contradiction. By $\mathcal{I S}(\ell+2,4), \mathcal{D}_{\ell+2}^{*, *}$ is an $\mathrm{H}^{*}(G, k)$-submodule of $\mathrm{H}^{*}\left(G, D^{*}\right)$ such that $\mathrm{H}^{*}\left(G, D^{*}\right) / \mathcal{D}_{\ell+2}^{*, *}$ is annihilated by $u_{\ell+2}$ which is a regular element on $\mathrm{H}^{*}\left(G, D^{*}\right)$. Thus $\mathcal{D}_{\ell+2}^{*, *}$ and also $E_{\ell+2}^{*, *}$ cannot be zero. This proves the theorem.

\section{An APPLiCATiON}

We present one easy application of the main theorem. There are numerous similar variations. As before assume that $k$ is a field of characteristic $p>0$.

Theorem 4.1. Suppose that $G$ is a finite group having an elementary abelian subgroup $E$ of p-rank 2. Let $\mathcal{S}$ be the collection consisting of all indecomposable projective $k G$-modules and all one dimensional $k G$-modules. If

$$
0 \longrightarrow C^{0} \stackrel{\partial}{\longrightarrow} C^{1} \longrightarrow \cdots \longrightarrow C^{\ell-1} \stackrel{\partial_{\ell-1}}{\longrightarrow} C^{\ell} \longrightarrow 0
$$

is a bounded exact sequence of $k G$-modules in $\operatorname{Add}(\mathcal{S})$, then $C^{*}$ is contractible.

Proof. Without loss of generality we may assume that $C^{*}$ has no nonzero direct summands other than itself. In particular, this means that $C^{\ell}$ is a sum of one dimensional modules. That is, if $C^{\ell}$ has a submodule $P$ that is projective, then $P$ is a direct summand of $C^{\ell}$ and $\partial_{\ell-1}$ followed by the projection on to $P$ splits. Thus, the complex $0 \rightarrow P \rightarrow P \rightarrow 0$ is a direct summand of $C^{*}$, violating our assumption. 
Next, we notice that the theorem is true if it holds in the case that $G$ is a $p$ group. Suppose that $Q$ is a Sylow $p$-subgroup of $G$. Assume that the restriction of $C^{*}$ to $Q$ is contractible. Then there is a $k Q$-homomorphism $\theta: C^{\ell} \rightarrow C^{\ell-1}$ such that $\partial_{\ell-1} \theta=\operatorname{Id}_{C^{\ell}}$. Let $\psi$ be the map $\psi=(1 /|G: Q|) \sum_{x \in G / Q} x \theta x^{-1}: C^{\ell} \rightarrow C^{\ell-1}$. Then $\psi$ is a $k G$-homomorphism that splits $\partial_{\ell-1}$.

We assume now that $G$ is a $p$-group and that each $C^{i}$ is a direct sum of trivial modules and a projective module. By Theorem 3.1 the restriction of $C^{*}$ to the elementary abelian subgroup $E$ is contractible. Hence, there is a $k E$-homomorphism $\theta: C^{\ell} \rightarrow C^{\ell-1}$ such that $\partial_{\ell-1} \theta=\operatorname{Id}_{C^{\ell}}$. Now write $C^{\ell-1}=M \oplus P$ where $M \cong k^{n}$ is a sum of trivial $k G$-modules and $P$ is a projective module. Let $\rho_{P}: C^{\ell-1} \rightarrow P$ be the projection, and $\iota_{P}$ be the inclusion of $P$ into $C^{\ell-1}$. Let $\rho_{M}$ and $\iota_{M}$ be the same for $M$ so that $\iota_{M} \rho_{M}+\iota_{P} \rho_{P}=\operatorname{Id}_{C^{\ell-1}}$. Notice that $\partial_{\ell-1} \iota_{P} \rho_{P} \theta$ is the zero map. The reason is that the image of $\theta$ is in the space of fixed points of $E$ on $P$, and since $P$ is a free module, this is a subset of the radical of $P$. Because $C^{\ell}$ is a sum of trivial modules, the radical of $P$ is in the kernel of $\partial_{\ell-1}$. It follows that $\partial_{\ell-1} \iota_{M} \rho_{M} \theta=\operatorname{Id}_{C^{\ell}}$. That is, $\psi=\iota_{M} \rho_{M} \theta$ splits $\partial_{\ell-1}$. To finish the proof we only need to notice that $\psi$ is a $k G$-homomorphism, since it is a linear map between sums of trivial modules.

Remark 4.2. The above theorem is not true for groups of $p$-rank one. This means groups whose Sylow $p$-subgroups are cyclic or quaternion. Indeed, for such a group the trivial module is periodic, and so there is a non-contractible exact complex beginning and ending with the trivial module, part of a projective resolution of the trivial module, with all intermediate modules projective.

\section{ACKNOWLEDGMENTS}

Both authors wish to thank Henning Krause and the University of Bielefeld for their hospitality and support during a visit when the initial ideas for this paper were developed. The authors would also like to thank the Newton Institute for Mathematical Sciences for support and hospitality during the programme "Groups, representations and applications: new perspectives," when part of the work on this paper was undertaken. Finally, they would like to thank the referee for taking the time to give us constructive feedback which helped improve the exposition.

\section{REFERENCES}

[1] Paul Balmer and Dave Benson, Resolutions by permutation modules, Arch. Math. (Basel) 115 (2020), no. 5, 495-498, DOI 10.1007/s00013-020-01493-w. MR4154564

[2] Glen E. Bredon, Introduction to compact transformation groups, Pure and Applied Mathematics, Vol. 46, Academic Press, New York-London, 1972. MR0413144

[3] Sören Illman, Smooth equivariant triangulations of $G$-manifolds for $G$ a finite group, Math. Ann. 233 (1978), no. 3, 199-220, DOI 10.1007/BF01405351. MR500993

[4] Daniel Quillen, Homotopy properties of the poset of nontrivial p-subgroups of a group, Adv. in Math. 28 (1978), no. 2, 101-128, DOI 10.1016/0001-8708(78)90058-0. MR493916

[5] Jeremy Rickard, Splendid equivalences: derived categories and permutation modules, Proc. London Math. Soc. (3) 72 (1996), no. 2, 331-358, DOI 10.1112/plms/s3-72.2.331. MR.1367082

[6] P. J. Webb, A local method in group cohomology, Comment. Math. Helv. 62 (1987), no. 1, 135-167, DOI 10.1007/BF02564442. MR882969

[7] P. J. Webb, Subgroup complexes, The Arcata Conference on Representations of Finite Groups (Arcata, Calif., 1986), Proc. Sympos. Pure Math., vol. 47, Amer. Math. Soc., Providence, RI, 1987, pp. 349-365, DOI 10.1016/0022-4049(82)90103-7. MR.933372 
[8] Clarence Wilkerson, A primer on the Dickson invariants, Proceedings of the Northwestern Homotopy Theory Conference (Evanston, Ill., 1982), Contemp. Math., vol. 19, Amer. Math. Soc., Providence, RI, 1983, pp. 421-434, DOI 10.1090/conm/019/711066. MR711066

Institute of Mathematics, Fraser Noble Building, University of Aberdeen, Aberdeen AB24 3UE, United Kingdom

Department of Mathematics, University of Georgia, Athens, Georgia 30602 